\title{
Anti-NMDAR Encephalitis in a 13-Year-Old Female: A 24-Month Clinical Follow-Up
}

\author{
Eunsil Kim, MD¹, Eu Gene Park, MD², Jiwon Lee, MD¹, Munhyang Lee, MD, PhD', \\ Jihye Kim, MD, $\mathrm{PhD}^{3}$, Jeehun Lee, MD, $\mathrm{PhD}^{1}$ \\ ${ }^{1}$ Department of Pediatrics, Samsung Medical Center, Sungkyunkwan University School of Medicine, Seoul; \\ ${ }^{2}$ Department of Pediatrics, Uijeongbu St. Mary's Hospital, The Catholic University School of Medicine, Seoul; \\ ${ }^{3}$ Department of Radiology and Center for Imaging Science, Samsung Medical Center, Sungkyunkwan University \\ School of Medicine, Seoul, Korea
}

\begin{abstract}
Anti-N-methyl-D-aspartate receptor (NMDAR) encephalitis is a rare autoimmune disorder manifesting as seizures, movement disorders, and psychiatric changes. However, there have been few case reports concerning this disorder in South Korean children. The current case report describes a pediatric patient with anti-NMDAR encephalitis. A 13-year-old female patient developed clonic movements of the right arm followed by aphasia, paresthesia, and right-sided hemiparesis. The electroencephalogram (EEG) results indicated electroclinical seizures arising from the left temporal area. Brain magnetic resonance imaging (MRI) revealed high signal intensity and cortical swelling in left temporal lobe. Anti-NMDAR antibodies were detected in the cerebrospinal fluid (CSF). The patient was treated with intravenous immunoglobulin and high-dose methylprednisolone and showed partial improvement in language skills, paresthesia, and motor power. The brain MRI and EEG results also indicated improvement. However, anti-NMDAR antibodies persisted in the CSF. After four doses of rituximab, the patient exhibited complete recovery of language and motor skills, and was seizure free under treatment with antiepileptic medication. There were no residual anti-NMDAR antibodies in the CSF at her 24-month follow-up visit. This case report elucidates the benefits of early intervention using rituximab to improve neurological deficits and achieve baseline recovery in patients with anti-NMDAR encephalitis. (2018;8:41-48)
\end{abstract}

Received April 13, 2018 Accepted June 25, 2018

Corresponding author:

Jeehun Lee, MD, PhD

Department of Pediatrics, Samsung Medica Center, Sungkyunkwan University School of Medicine, 81 Irwon-ro, Gangnam-gu, Seoul 06351, Korea

Tel. +82-2-3410-0910

Fax. $+82-2-3410-0043$

E-mail; jhlee0101@skku.edu
Key words: Autoimmune, Encephalitis, Child, NMDA receptor antibody

\section{Introduction}

Anti-N-methyl-D-aspartate receptor (NMDAR) encephalitis is a well-described clinical disorderin children and adults, ${ }^{1}$ and is one of the well-known contributors to encephalitis in children. ${ }^{2}$ This autoimmune disease is becoming increasingly recognized in the pediatric population, ${ }^{3}$ yet the pathophysiology in most cases of anti-NMDAR encephalitis remains unknown. However, a few cases of this disorder have been thought to have a paraneoplastic origin. ${ }^{4,5}$ Children with anti-NMDAR encephalitis initially present with prodromal symptoms of neuropsychiatric abnormalities in behavior, speech, and mood, which are often accompanied by personality change, memory loss, and seizures. A definitive diagnosis requires the detection of NMDAR antibodies in the cerebrospinal fluid (CSF). ${ }^{6}$ Early diagnosis and prompt treatment can improve outcomes; however, there is no consensus guideline for the optimal management of patients with this disease, particularly in children. ${ }^{6,7}$ Here, we report a case of anti-NMDAR encephalitis in a 13-year-old female treated with rituximab. This case report may contribute towards elucidating the benefits of early intervention using rituximab to improve neurological deficits, reduce the amount of residual anti-NMDAR antibodies in the CSF, and achieve baseline recovery in patients with anti-NMDAR encephalitis, who show only a partial response or are refractory to first-line therapies.

\section{Case}

A 13-year-old female was referred to Samsung Seoul Hospital with frequent clonic movements of the right arm and leg followed by right-sided weakness, paresthesia, and acute onset of aphasia. One month prior, she had clonic movements of the right arm and leg last- 
ing about 2 minutes without loss of consciousness. The results of electroencephalography (EEG) and brain magnetic resonance imaging (MRI) were unremarkable at initial presentation. She was prescribed levetiracetam for the prevention of seizures.

Two weeks after the initial presentation, the patient experienced right leg weakness along with right hand and right foot paresthesia and was referred to Samsung Seoul Hospital. Upon admission, the patient experienced fever and her temperature had risen to $38^{\circ} \mathrm{C}$. She exhibited clonic movements of the right arm, was inarticulate and could neither verbalize nor understand others.

She was born from non-consanguineous parents at the 38th week of gestation by spontaneous vaginal delivery at an appropriate gestational age. There was no history of significant illness, the results of her general physical examination were normal, and there was no evidence of an external injury. She was alert and the results of her cranial nerve examination were also normal. She exhibited decreased right-sided motor power (motor grade IV) and right-sided paresthesia. There were no pathological reflexes.

\section{Initial clinical course}

The patient exhibited continuous right-sidedclonic movements at the time of transfer. The clinical impression was an epilepsiapartialis continua because the EEG revealed continuous slow waves in the left hemisphere and frequent spikes or sharp wave discharges from the left temporal areas and identified multiple electroclinical seizures arising from the left temporal area (Fig. 1). The seizures werecontrolled with intravenous lorazepam (5 mg). She was admitted to the general ward and treated with antibiotics (cefotaxime, vancomycin, and azithromycin), an antiviral agent (acyclovir), antiepileptic drugs (levetiracetam and oxcarbazepine), and dexamethasone.

\section{Laboratory findings}

The complete blood count was normal. Antinuclear antibodies, rheumatoid factor, immunoglobulins, complement, thyroid function test, and $\alpha$-fetoprotein were within normal limits. There was no remarkable elevation of anti-viral antibody titers from serum and CSF samples, including herpes simplex virus, enterovirus, and Japanese B encephalitis virus. The CSF analysis did not indicate pleocytosis or other biochemical abnormalities and was negative for cultures of bacteria and viruses (Table 1). The initial EEG demonstrated electroclinical seizures arising from the left temporal area. The follow-up EEG revealed intermittent and generalized slow waves, which per- tains to diffuse cerebral dysfunction (Fig. 1). T2-weighted brain MRI revealed multifocal high signal intensity lesions and cortical swelling involving bilateral temporal lobes, including the limbic system, which were more promient in the left hemisphere (Fig. 2).

\section{Clinical course during initial admission}

The patient was empirically treated for acute bacterial meningitis and acute encephalitis with intravenous vancomycin $(60 \mathrm{mg} / \mathrm{kg} / \mathrm{day}$ divided in 4 doses), cefotaxime (160 mg/kg/day divided in 4 doses), and acyclovir (30 mg/kg/day divided in 3 doses) until the tests for microorganisms yielded results. Additionally, azithromycin (10 mg/kg/day) was administered because mycoplasma pneumoniae infection was suspected, as the mycoplasma pneumoniae antibody titer was 1:640. For the treatment of seizures, diphenylhydantoin $(4 \mathrm{mg} / \mathrm{kg} / \mathrm{day})$ and levetiracetam (20 mg/kg/day) were administered. From the $2 \mathrm{nd}$ hospital day, intravenous immunoglobulin (400 mg/kg/day for 5 days) was introduced considering the differential diagnoses (limbic encephalitis, viral encephalitis, autoimmune encephalitis, and acute disseminated encephalomyelitis) based on brain MRI results. On the 10th hospital day, antibiotic treatments were ceased because the bacterial cultures exhibited no growth of bacterial organisms. Despite the negative polymerase chain reaction results for herpes simplex virus (HSV1 and HSV2), acyclovir was continued for 14 days for the complete treatment of possible herpes virus encephalitis.

On the 15th hospital day, the CSF tests for autoimmune antibodies revealed the presence of anti-NMDAR antibodies (Table 1). Following this result, methylprednisolone pulse therapy was commenced at $790 \mathrm{mg}$ once daily (15 mg/kg/day) for three consecutive days. To determine the etiology of the anti-NMDAR encephalitis, particularly a possible paraneoplastic origin, chest and abdomen-pelvis computed tomography (CT) scans were performed. On abdomen-pelvis $\mathrm{CT}$, a small, low, attenuated lesion was noted in the right adnexa. For further evaluation, an abdomen-pelvis MRI was performed; the lesion was concluded to be multiple follicles of both ovaries, and there was no demonstrable tumorous lesion (Fig. 3).

Between the 21st and 25th hospital day, there was a significant improvement in the patient's condition regarding speech, motor power, and paresthesia. The lesions observed on brain MRI decreased in extent, but high signal intensity with cortical swelling involving the temporal lobes and the limbic system, predominantly in the left hemisphere, was still observed on fluid-attenuated inversion 
A

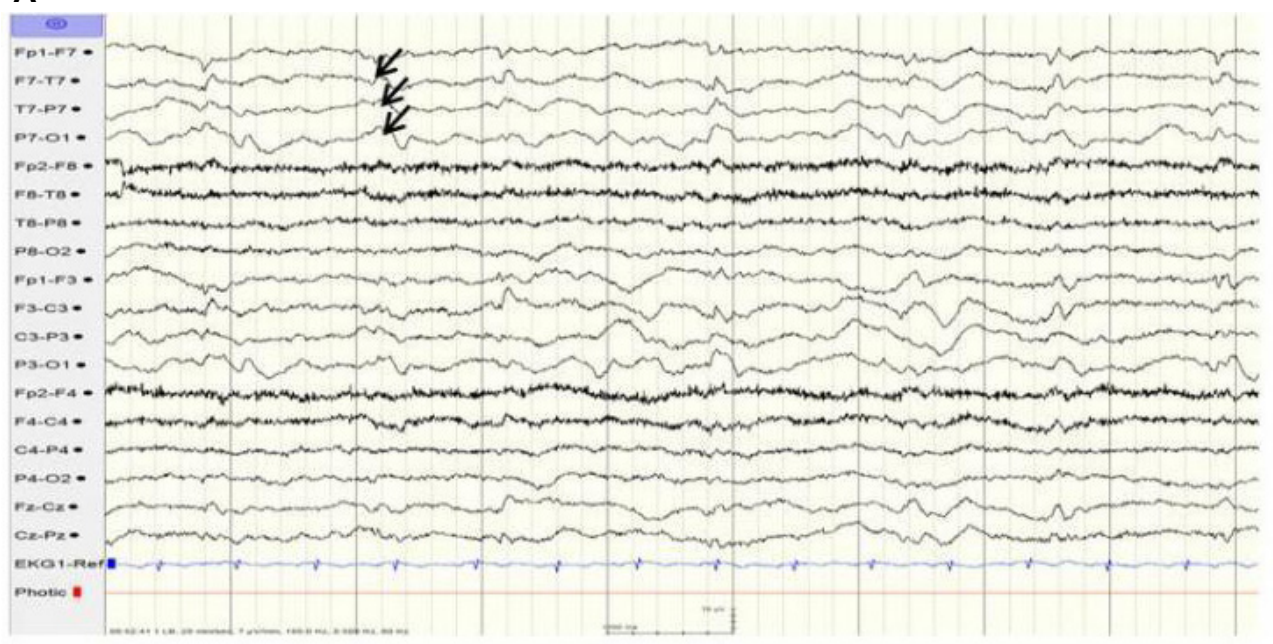

B

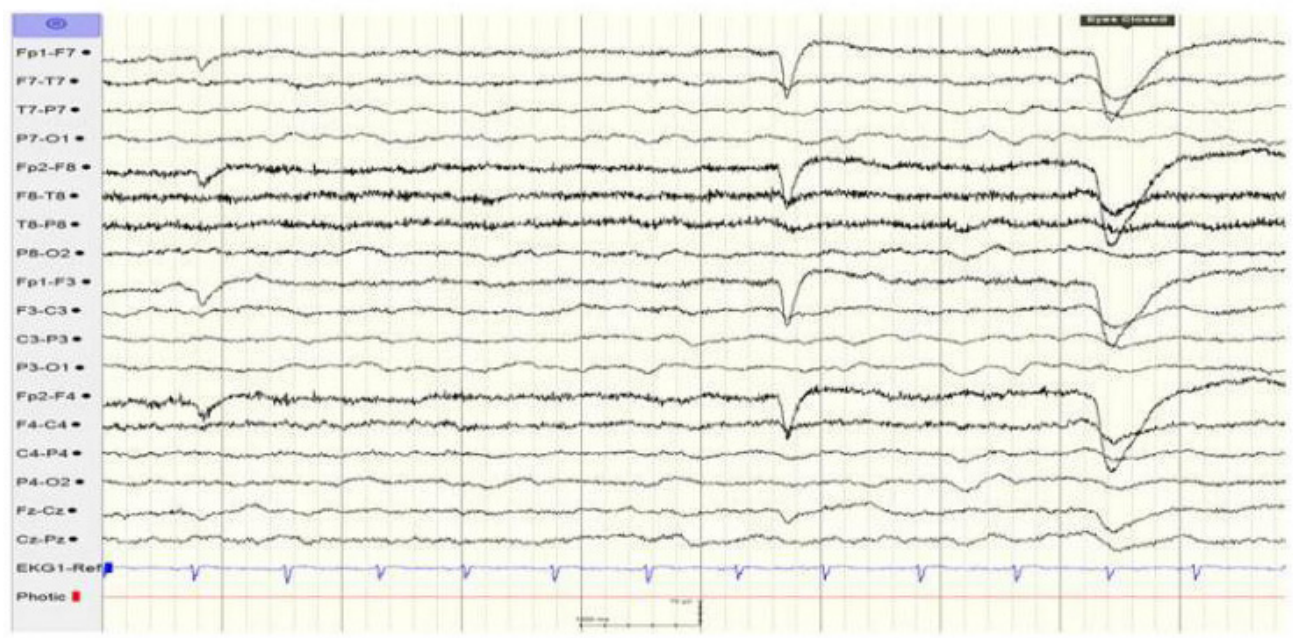

\section{C}

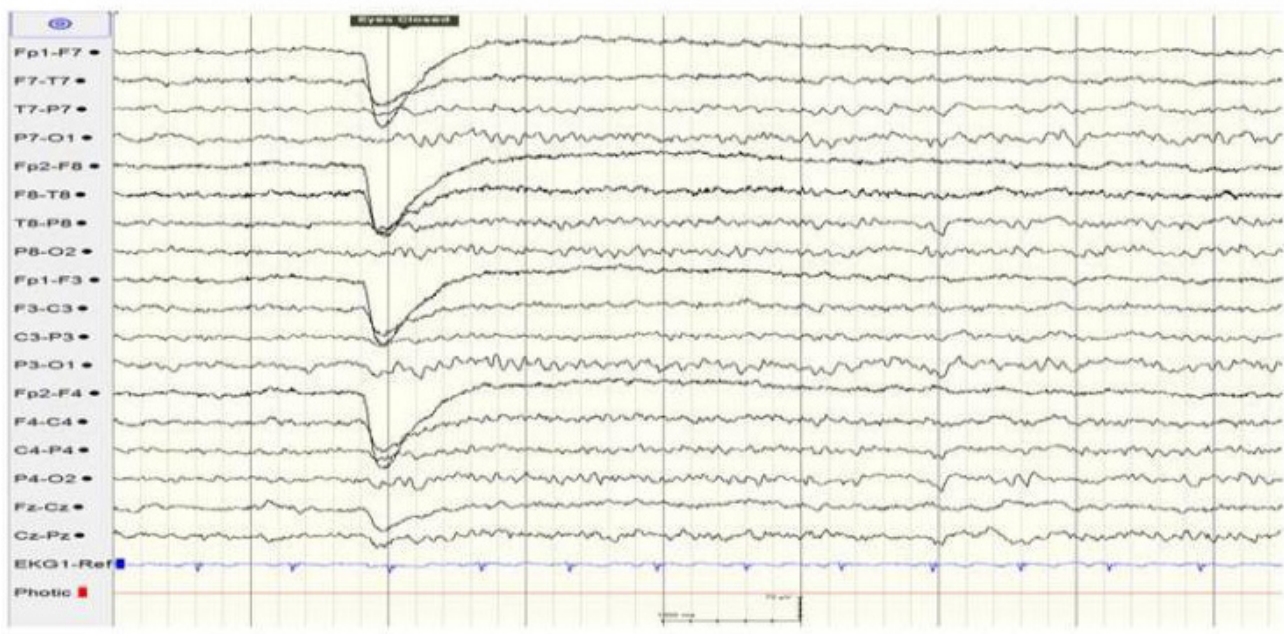

Figure 1. (A) One month after initial presentation (on admission): regional sharp waves from the left temporal lobe (arrows) and cerebral dysfunction in the left hemisphere. (B) Follow-up EEG after methylprednisolone pulse therapy: diffuse or severe regional cerebral dysfunction in the left fronto-centro-temporal areas; no epileptic discharges. (C) Follow-up EEG after rituximab therapy: diffuse cerebral dysfunction in the left fronto-centro-temporal or left temporo-parieto-occipital area; no epileptic discharges. EEG, electroencephalogram. 
Table 1. Results of laboratory tests

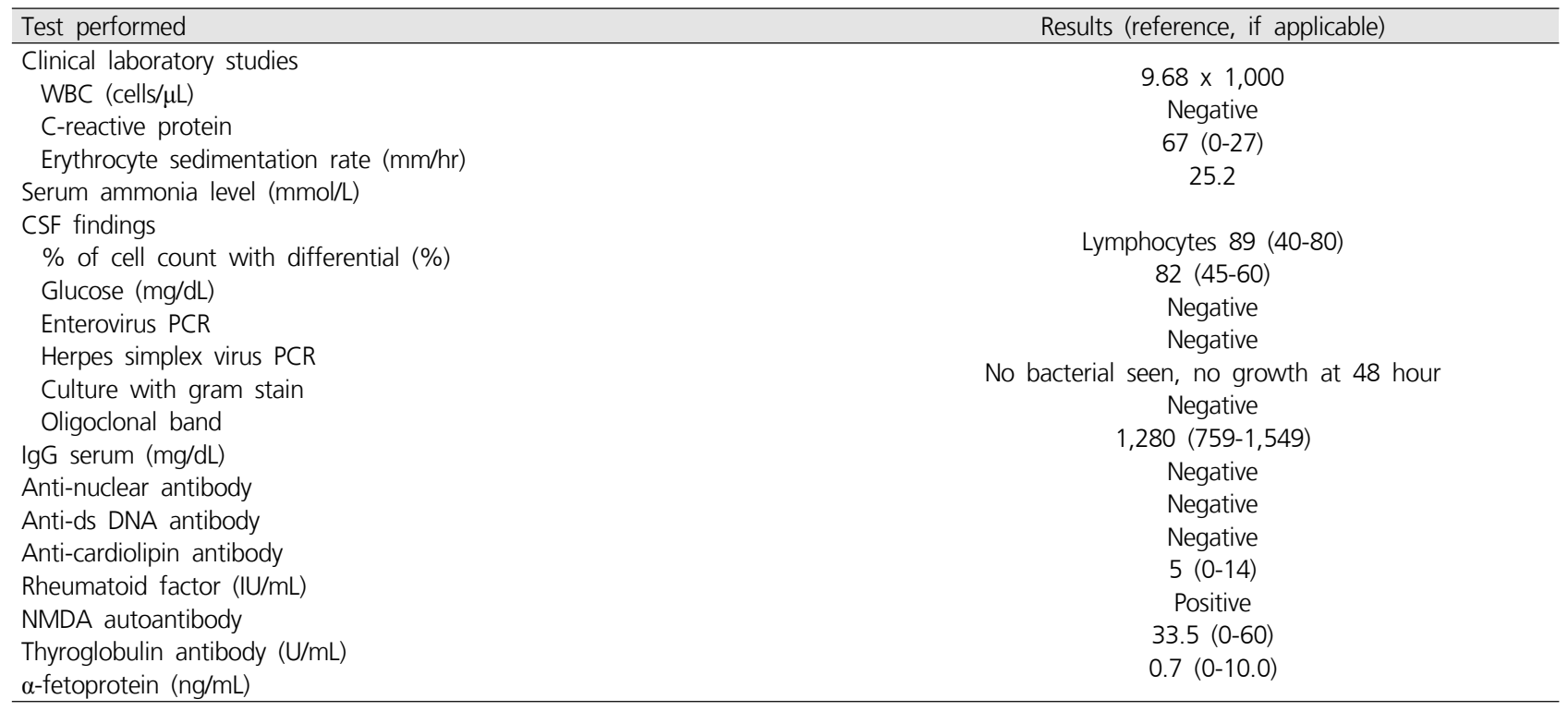

WBC, white blood cell; CSF, cerebrospinal fluid; PCR, polymerase chain reaction; NMDA, N-methyl-D-aspartate receptor.
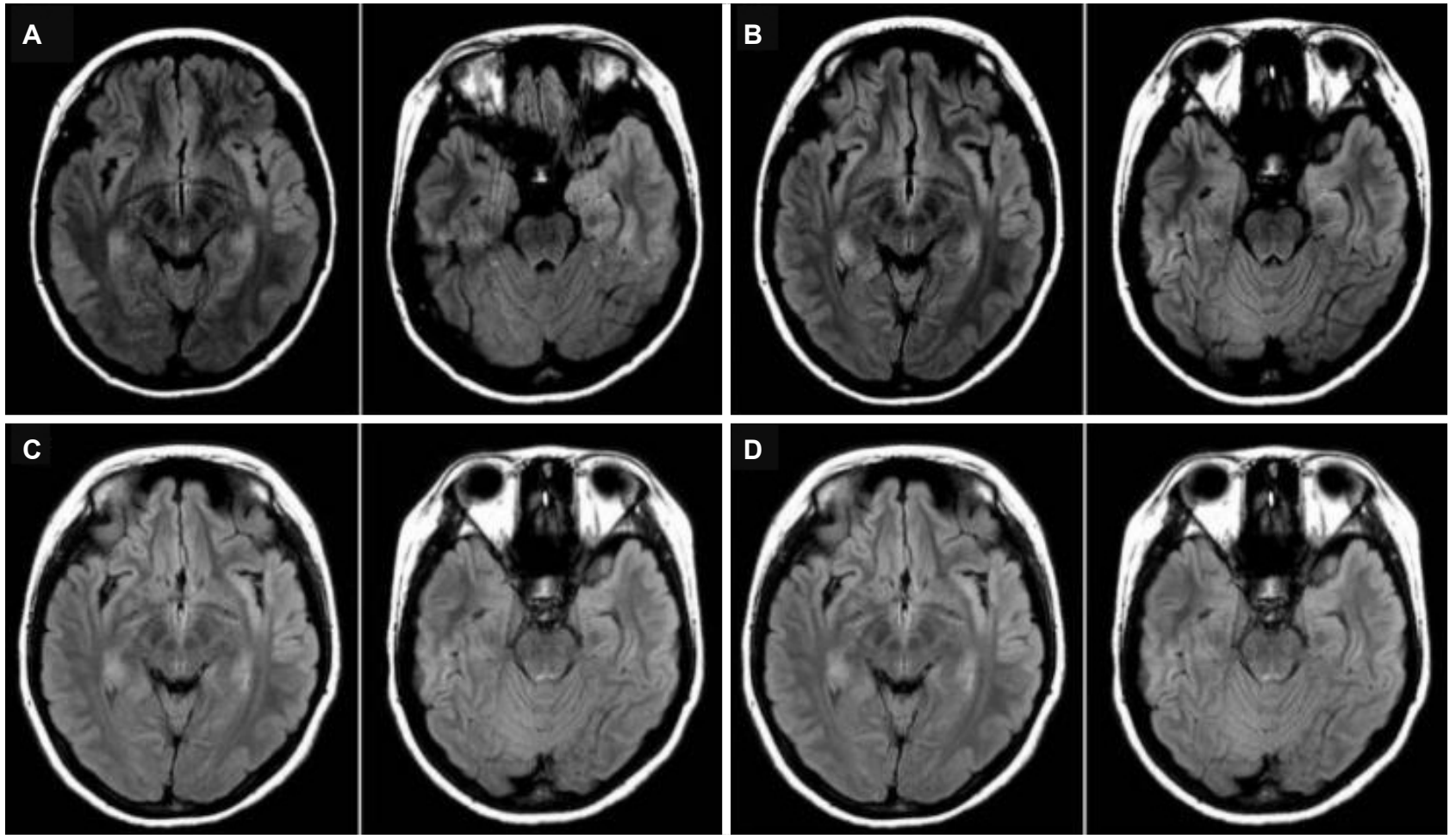

Figure 2. Magnetic resonance imaging (MRI) of the brain. (A) Initial brain MRI: FLAIR hyperintensities and cortical swelling involving the temporal lobe, including the limbic system, predominantly on the left side. (B) Follow-up brain MRI after 14 days in the hospital: improved interval. (C) Follow-up brain MRI after 2 months in the hospital: no interval change. (D) Follow-up brain MRI after 9 months following rituximab therapy: no interval change. FLAIR, fluid-attenuated inversion recovery. 

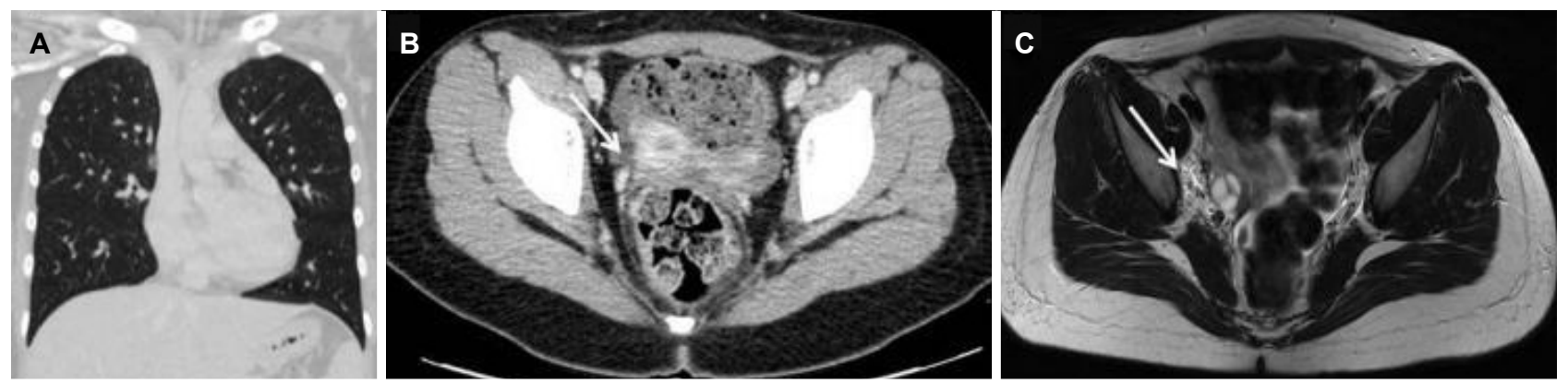

Figure 3. Evaluation of anti-NMDAR encephalitis etiology. (A) There was no remarkable tumorous condition in the chest CT. (B) Abdomen CT: 1 x $0.5 \mathrm{~cm}$ hypodense lesion in the right adnexa (arrow). (C) Abdomen-pelvis MRI: multiple follicles of both ovaries without a demonstrable tumorous lesion (arrow). NMDAR, N-methyl-D-aspartate receptor; CT, computed tomography; MRI, magnetic resonance imaging.

recovery (FLAIR). The follow-up EEG on the 21st hospital day revealed continuous slow waves in the left fronto-centro-temporal areas without epileptiform discharges.

The patient was discharged on the 25th hospital day and was prescribed clobazam, valproic acid, diphenylhydantoin, and oxcarbazepine. The patient's receptivity to communication appeared to be improved, but her speech, particularly word variety, sentence length, and word-finding ability, remained slightly below baseline.

\section{Clinical follow-up after initial admission}

By the time of the outpatient follow-up visit, approximately one month after discharge, the patient showed near complete resolution of sensory aphasia, motor weakness, and paresthesia symptoms and a partial resolution of calculation disability and motor aphasia symptoms. However, anti-NMDAR antibodies were still present in the CSF (weakly positive antibody reactivity). Four months after discharge, despite the nearly complete resolution of clinical symptoms, there were no remarkable improvements in brain MRI and EEG results, i.e., no interval change inresidual high signal intensity lesions involving the left temporal lobe, including the limbic system, on brain MRI; and generalized continuous slow waves onthe EEG. As a second-line therapy to treat the persistent presence of anti-NMDAR antibodies in the CSF, rituximab (a monoclonal anti-CD20 antibody), $375 \mathrm{mg} / \mathrm{m}^{2}$ per dose weekly was administered four times in total. Eight months after discharge, the patient achieved symptom-free status. The EEG revealed localized intermittent slow waves in the left temporal areas, and the brain MRI indicated decreased extents of high signal intensities in the left temporal lobe (Fig. 2). She was suspected to be at risk of a relapse based on the EEG and the brain MRI results, and a third CSF study was conductedbecause changesinanti-NMDAR antibody titers in the CSF correlate with clinical relapses. $^{8}$

At the last visit (24 months after symptom onset and 18 months after rituximab treatment), the patient maintained a seizure-free state and performed well in school, with no demonstrable tumor lesion in the follow-up tumor screening. Therefore, the remaining antiepileptic drug, oxcarbazepine, was discontinued.

\section{Discussion}

Anti-NMDAR encephalitis, originally described in 2005 as a paraneoplastic syndrome in a group of women with ovarian teratoma, was linked to the presence of anti-NMDAR antibodies and was officially defined in $2007 .{ }^{5}$ Approximately $40 \%$ of reported patients with anti-NMDAR encephalitis are $<18$ years of age. ${ }^{6}$ A recent study compiling data from more than 577 patients with anti-NMDAR encephalitis found that $37 \%$ of them were in the pediatric age group. ${ }^{9}$ Most children with anti-NMDAR encephalitis present with seizures or movement disorders ${ }^{10}$ rather than psychiatric symptoms such as anxiety, agitation, paranoia, and visual or auditory hallucinations, which are more predominant in adults with anti-NMDAR encephalitis. $^{5,11}$

The patient in this study presented with epilepsia partialis continua, which is rarely reported in pediatric anti-NMDAR encephalitis, $^{12,13}$ along with motor aphasia and unilateral sensory changes. According to a recent study, the first neurological symptoms observed in young children with anti-NMDAR encephalitis included seizure $(72 \%)$, particularly focal seizure ( $42 \%)$, with a median onset of 15 days before other encephalitis symptoms appeared; other patients mostly exhibited behavioral disorders $(26 \%){ }^{14}$ Thus, in patients presenting a novel onset of altered mental status, seizures, or behavioral changes, anti-NMDAR encephalitis should be systemati- 
cally considered in the absence of other apparent etiologies.

With early diagnosis and effective treatment, patients with anti- NMDAR encephalitis have a relatively good prognosis and can achieve baseline recovery and complete resolution at follow-up examination. ${ }^{4,15}$ According to the California Encephalitis Project, the number of young patients with anti-NDMAR encephalitis was greater than that of patients with viral encephalitis. ${ }^{16}$ Thus, anti-NMDAR encephalitis must be considered and should be part of the differential diagnosis in young patients with limbic encephalitis.

In this case, the EEG revealed regional epileptiform discharges and slow waves, which are frequently described in children with antiNMDAR encephalitis as well as in adults. ${ }^{17}$ This patient initially presented unremarkable changes in the brain MRI, but the following MRI (after the presentation of motor aphasia and epilepsiapartialis continua) indicated increased signals on T2-weighted FLAIR MRI sequences involving the temporal lobes, including the limbic system, predominantly in the left hemisphere. The findings of the brain MRI in our patient were consistent with findings of anti-NMDAR encephalitis. ${ }^{12,18}$ In most cases (89\%), brain MRI results are normal or reveal non-specific focal changes at the initial presentation, as in this case. ${ }^{6}$ Another study reported that most patients with NMDAR encephalitis (66\%) had normal brain MRI findings, and the remaining $44 \%$ had a wide variation in the distribution and degree of T2-FLAIR hyperintense sig- nal changes throughout the brain. ${ }^{18}$

The majority of anti-NMDAR encephalitis cases are idiopathic in pathophysiology; however, a significant minority can be attributed to a paraneoplastic origin. ${ }^{19}$ Regardless of tumor presence, patients with anti-NMDAR encephalitis should be treated with first-line immunotherapy, typically corticosteroids, intravenous immunoglobulin (IVIG), or plasmapheresis. The patients without tumors have an initial response rate of $48 \%{ }^{4}$ In pediatric patients, first-line immunotherapy is effective in only half of the patients diagnosed with anti-NMDAR encephalitis because pediatric patients are less likely to have causative tumors compared to adults. ${ }^{20}$ If there is no response to the first-line immunotherapy, rituximab or cyclophosphamide are usually recommended for the treatment of clinical symptoms and prevention of relapse. $4,5,10,20,21$

In this patient, the anti-NMDAR antibodies in the CSF were present without a causative tumor. After detection of anti-NMDAR antibodies in the CSF, methylprednisolone pulse therapy was added to the initial IVIG treatment considering the incomplete resolution of the clinical symptoms. Although remarkable clinical improvements were observed after high-dose steroid treatment, there were residual anti-NMDAR antibodies in the CSF. After the additional rituximab therapy, the patient no longer reported any symptoms (Fig. 4). Despite a prolonged presence of abnormalities in the brain MRI and

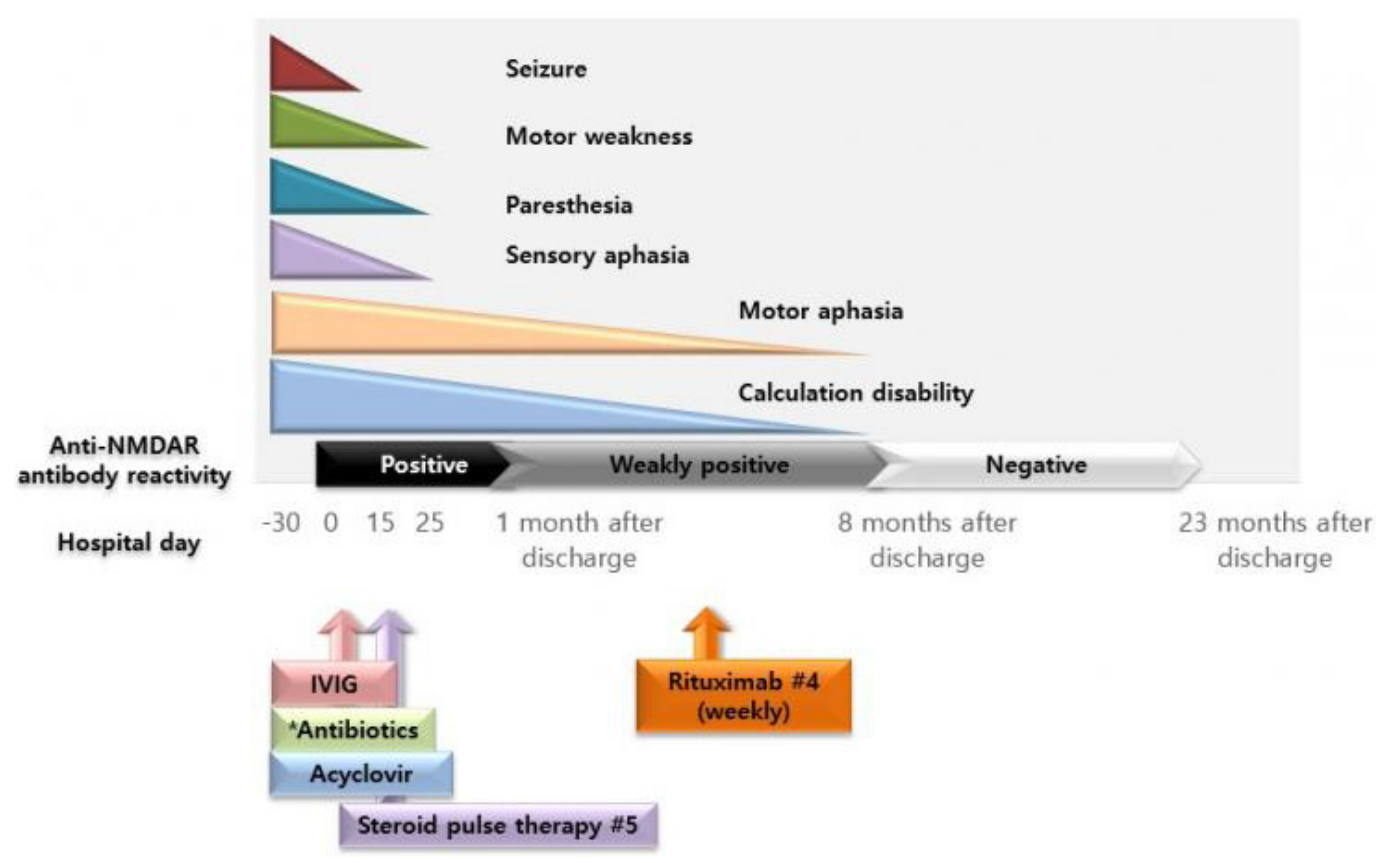

Figure 4. Clinical course of this patient—a 13-year-old female diagnosed with anti-NMDAR encephalitis. NMDAR, N-methyl-D-aspartate receptor; IVIG, intravenous immunoglobulin. *Antibiotics: vancomycin, cefotaxime, azithromycin. 
EEG, there was no demonstrable tumor lesion in the follow-up tumor screening test. A CSF study was performed because changes in anti-NMDAR antibody titers in the CSF correlate with clinical relapses, ${ }^{8}$ and anti-NMDAR antibodies were not present in the patient's CSF.

Among patients with anti-NDMAR encephalitis who do not respond to first-line therapy (i.e., steroids, intravenous immunoglobulin), those receiving second-line immunotherapy (i.e., rituximab) achieve a better outcome than those who do not receive second-line agents. ${ }^{7}$ Patients receiving second-line immunotherapy also experience fewer relapses than those not receiving such therapy. ${ }^{9}$ Accumulating data suggest that rituximab is an effective treatment for anti-NMDAR encephalitis in both children and adults. ${ }^{22}$

Anti-NMDAR-encephalitis is mediated by immunoglobulin $\mathrm{G}$ antibodies against the GluN1 subunit of the neuronal NMDA receptor. The inflammatory neuronal dysfunction is thought to be initially reversible but potentially progresses to permanent neuronal destruction if untreated, due to prolonged inflammation and NMDA-mediated glutamate excitotoxicity. ${ }^{18,23}$ Thus, rituximab may be a favorabletreatment option for patients who have residual anti-NMDAR antibodies in the CSF and for those who do not respond to immunosuppressive therapy (i.e., corticosteroids andIVIG). ${ }^{7,24}$

In summary, this report discusses the case of a 13-year-old female with anti-NMDAR encephalitis, which was idiopathic in etiology. She received first-line (i.e., steroid and intravenous immunoglobulin) and second-line (i.e., rituximab) immunotherapy, and 18 months after initial presentation, normalcerebral function was restored and there wereno residual anti-NMDAR antibodies in the CSF. Patients with anti-NMDAR encephalitis typically present with psychiatric symptoms and partial seizures, and it is important that they receivean early diagnosis and prompt treatment for an improved prognosis. The medical history, combined with brain MRI, EEG, and CSF examination, was helpful in the diagnosis of anti-NMDAR encephalitis. Our case suggests that in patients who have residual anti-NMDAR antibodies or in those who do not tolerate first-line immunotherapy, rituximab could be considered as a treatment option. Thus, physicians might consider the early initiation of second-line immunotherapy in certain cases of anti-NMDAR encephalitis.

\section{References}

1. DeSena AD, Noland DK, Matevosyan $K$, et al. Intravenous methylprednisolone versus therapeutic plasma exchange for treatment of anti-N-methyl-D-aspartate receptor antibody encephalitis: a retrospective review. J Clin Apher 2015;30:212-6.

2. Dale RC, Gorman MP, Lim M. Autoimmune encephalitis in children: clinical phenomenology, therapeutics, and emerging challenges. Curr Opin Neurol 2017;30:334-44.

3. Zhu WW, Liao WP, Yi YH, Song XW. Efficacy and safety of cyclophosphamide as a sequential immunotherapy drug for anti-N-methyl-D-aspartate receptor encephalitis in children. Zhongguo Dang Dai Er Ke Za Zhi 2017;19:668-71.

4. Dalmau J, Lancaster E, Martinez-Hernandez E, Rosenfeld MR, Balice-Gordon R. Clinical experience and laboratory investigations in patients with anti-NMDAR encephalitis. Lancet Neurol 2011;10:63-74.

5. Dalmau J, Gleichman AJ, Hughes EG, et al. Anti-NMDA-receptor encephalitis: case series and analysis of the effects of antibodies. Lancet Neurol 2008;7:1091-8.

6. Irani SR, Bera K, Waters $P$, et al. N-methyl-D-aspartate antibody encephalitis: temporal progression of clinical and paraclinical observations in a predominantly non-paraneoplastic disorder of both sexes. Brain 2010;133:1655-67.

7. Hallowell S, Tebedge E, Oates M, Hand E. Rituximab for Treatment of Refractory Anti-NMDA Receptor Encephalitis in a Pediatric Patient. J Pediatr Pharmacol Ther 2017;22:118-23.

8. Gresa-Arribas N, Titulaer MJ, Torrents A, et al. Antibody titres at diagnosis and during follow-up of anti-NMDA receptor encephalitis: a retrospective study. Lancet Neurol 2014;13:167-77.

9. Goldberg EM, Titulaer $M$, de Blank PM, Sievert A, Ryan N. Anti-N-methyl-D-aspartate receptor-mediated encephalitis in infants and toddlers: case report and review of the literature. Pediatr Neurol 2014;50:181-4.

10. Lin JJ, Lin KL, Hsia SH, et al. Anti-N-methyl-D-aspartate receptor encephalitis in Taiwan--a comparison between children and adults. Pediatr Neurol 2014;50:574-80.

11. Brenton JN, Kim J, Schwartz RH. Approach to the management of pediatric-onset anti-N-Methyl-d-Aspartate (anti-NMDA) receptor encephalitis: a case series. J Child Neurol 2016;31:1150-5.

12. Kim EH, Kim YJ, Ko TS, Yum MS, Lee JH. A young child of anti-NMDA receptor encephalitis presenting with epilepsia partialis continua: the first pediatric case in Korea. Korean J Pediatr 2016;59(Suppl 1):S133-8.

13. Kumakura A, Miyajima T, Fujii T, Takahashi Y, Ito M. A patient with epilepsia partialis continua with anti-glutamate receptor epsilon 2 antibodies. Pediatr Neurol 2003;29:160-3.

14. Favier $M$, Joubert $B$, Picard $G$, et al. Initial clinical presentation of young children with $\mathrm{N}$-methyl-d-aspartate receptor encephalitis. Eur J Paediatr Neurol 2018;22:404-11.

15. Nunez-Enamorado N, Camacho-Salas A, Belda-Hofheinz S, et al. Fast and spectacular clinical response to plasmapheresis in a paediatric case of anti-NMDA encephalitis. Rev Neurol 2012;54:420-4.

16. Gable MS, Sheriff H, Dalmau J, Tilley DH, Glaser CA. The frequency of autoimmune $\mathrm{N}$-methyl-D-aspartate receptor encephalitis surpasses that of individual viral etiologies in young individuals enrolled in the 
California Encephalitis Project. Clin Infect Dis 2012;54:899-904.

17. Huang $Q$, Wu $Y$, Qin $R$, Wei $X$, Ma M. Clinical characteristics and outcomes between children and adults with anti-N-Methyl-D-Aspartate receptor encephalitis. J Neurol 2016;263:2446-55.

18. Kelley BP, Patel SC, Marin HL, Corrigan JJ, Mitsias PD, Griffith B. Autoimmune encephalitis: pathophysiology and imaging review of an overlooked diagnosis. AJNR Am J Neuroradiol 2017;38:1070-8.

19. Armangue T, Titulaer MJ, Málaga I, et al. Pediatric anti-N-methyl-D-aspartate receptor encephalitis-clinical analysis and novel findings in a series of 20 patients. J Pediatr 2013;162:850-6.e2.

20. Titulaer MJ, McCracken L, Gabilondo I, et al. Treatment and prognostic factors for long-term outcome in patients with anti-NMDA receptor encephalitis: an observational cohort study. Lancet Neurol
2013;12:157-65.

21. Salvucci $A$, Devine IM, Hammond $D$, Sheth RD. Pediatric anti-NMDA (N-methyl D-aspartate) receptor encephalitis. Pediatr Neurol 2014;50: 507-10.

22. Bien $C G$, Vincent $A$, Barnett $M H$, et al. Immunopathology of autoantibody-associated encephalitides: clues for pathogenesis. Brain 2012; 135:1622-38.

23. Bravo-Oro A, Acosta-Yebra D, Grimaldo-Zapata IP, Reyes-Vaca G. Reversible cortical atrophy secondary to anti-NMDA receptor antibody encephalitis. Rev Neurol 2015;60:447-52.

24. lizuka T, Ishima D, Kaneko J, Nishiyama K. Clinical spectrum and treatment strategy in anti-NMDA receptor encephalitis: current status and issues. Rinsho Shinkeigaku 2014;54:1098-102. 